\title{
A GSH-Responsive Nanoprodrug System Based on Self-Assembly of Lactose Modified Camptothecin for Targeted Drug Delivery and Combination Chemotherapy
}

This article was published in the following Dove Press journal:

International Journal of Nanomedicine

\section{Chenxi Hou',* \\ Ning $\mathrm{Ma}^{\mathrm{l}, *}$ \\ Ziyan Shen' \\ Guanyu Chi ${ }^{1}$ \\ Shuang Chao' \\ Yuxin Pei ${ }^{1}$ \\ Lan Chen ${ }^{2}$ \\ Yuchao $\mathrm{Lu}^{2}$ \\ Zhichao Pei ${ }^{1}$}

'Shaanxi Key Laboratory of Natural Products \& Chemical Biology, College of Chemistry and Pharmacy, Northwest A\&F University, Yangling, Shaanxi 7I2100, People's Republic of China; ${ }^{2}$ Analysis Center of College of Science \&

Technology, Hebei Agricultural

University, Huanghua, Hebei 06II00,

People's Republic of China

*These authors contributed equally to this work

\begin{abstract}
Background: Conventional chemotherapy using small molecular antitumor drugs suffers from several limitations, for instance poor water solubility, high toxicity, and lack of specificity. However, prodrugs constructed by covalent modification of anticancer drugs can overcome these limitations, which are able to release its active form after entering the tumor tissues by specific stimulus response.
\end{abstract}

Methods: A GSH-responsive glyco-nanoprodrug system has been constructed by selfassembled of amphiphilic lactosemodified camptothecin prodrug molecular (Lac-SS-CPT) for targeting drug delivery and combination therapy.

Results: Using HL7702 cells as experimental models, the cytotoxic effects of Lac-SS-CPT were investigated to $10-30 \mu \mathrm{mol} / \mathrm{L}$ for 48 hours. Notably, the cell viability of Lac-SS-CPT to HL7702 cells was higher compared with free CPT which indicated that Lac-SS-CPT can reduce side-effects. Simultaneously, we have evaluated the anticancer efficiency of doxorubicin hydrochloride (DOX)-loaded Lac-SS-CPT glyco-nanoprodrug system (Lac-SS-CPT @DOX), where Lac-SS-CPT@DOX and free DOX incubated with HpeG2 cells and HL7702 cells for 24, 48, and 72 hours, respectively. It turned out that Lac-SS-CPT@DOX encapsulated anticancer drug (DOX) could decrease DOX side-effect on HL7702 cells and increase DOX anticancer efficiency. More importantly, the CPT and DOX were released from Lac-SS -CPT@DOX in HepG2 cells where a higher GSH concentration exists. Moreover, combination therapy efficiency was evaluated, where free DOX and Lac-SS-CPT@DOX incubated with DOX-resistance HepG2 cells (HepG2-ADR cells), respectively.

Conclusion: The results revealed that the Lac-SS-CPT@DOX could enhance the cytotoxicity of DOX for HepG2-ADR cells and provided a new idea for designing an advanced nanoprodrug system toward combination therapy.

Keywords: nanoprodrug, target, GSH-responsive, drug delivery, combination chemotherapy

\section{Introduction}

Nowadays, chemotherapy remains the most significantly important therapy for various types of cancer which was used in combination with surgical treatment. ${ }^{1-7}$ However, conventional chemotherapy, which uses small molecular anticancer drugs, also suffers from several limitations, for instance poor water solubility, high toxicity, and lack of specificity. ${ }^{8-13}$ In order to solve these problems, a clinically proven strategy, which is to construct prodrugs by covalent modification of anticancer drugs, has been developed,
Correspondence: Zhichao Pei; Yuchao Lu Tel +862987091196

Fax +86 2987092769

Email peizc@nwafu.edu.cn;

luyuchao@hebau.edu.cn
International Journal of Nanomedicine 2020:15 10417-10424

10417

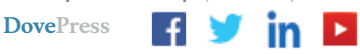

http://doi.org/10.2147un 5276470 
where the drugs can release their active form after entering the tumor tissues by specific stimulus response. ${ }^{14-21}$ Many drugs contain active groups, such as hydroxyls and amines, that can be modified by simple chemical means and have laid a foundation for the construction of prodrugs. Among them, the nanoprodrug system as a novel prodrug system attracted much attention due to their good stability, negligible drug leakage during circulation, improved anticancer drug loading efficiency, and control of drug release in tumor tissues. ${ }^{22-26}$ Especially, the nanoprodrug system has recently been used for drug co-delivery with successfully achieved combined chemotherapeutic effects, which provided a new way to overcome the resistance of tumor cells. ${ }^{27,28}$

It is well known that camptothecin (CPT), which inhibits topoisomerase I activity, has significant anticancer activity against various solid tumors in vitro. ${ }^{29,30}$ However, the major problem encountered with CPT is its extremely low solubility and side effects, which lead to the failure of clinical treatment. ${ }^{31}$ In order to overcome the problem, CPT usually forms prodrug by linking to different hydrophilic moieties such as peptides, ${ }^{32}$ oligomer ethylene glycol, ${ }^{33-37}$ or drug molecules. ${ }^{11,38}$ Compared with these hydrophilic moieties, carbohydrates not only have excellent biocompatibility owing to the hydrophilic motif, but also possess the unique ability to target cancer cells on account of the specific interactions between carbohydrate and protein. ${ }^{39}$ In 2015, D’Souza and Devarajan ${ }^{40}$ reported that asialoglycoprotein which can improve the internalization of galactose/lactose by carbohydrate-protein interaction was overexpressed on the surface of the HepG2 cells membrane. So, nanoprodrugs modified with galactose/lactose could be constructed to realize the purpose of targeting HepG2 cells to improve drug delivery efficiency and reduce the side effect.

Herein, we fabricated a novel amphiphilic lactosecamptothecin prodrug molecular Lac-SS-CPT which can self-assemble into Lac-SS-CPT glyco-nano prodrug system for GSH responsive and targeted drug co-delivery due to the existence of disulfide bond and lactose. ${ }^{41-44}$ Then, the CPT-based glyco-nano prodrug system further provides a suitable environment for entrapping another anticancer drug DOX for combination therapeutic effects (see Scheme 1). ${ }^{45}$

\section{Materials and Methods}

\section{Materials and Instrumentations}

All of the reagents and solvents used in this paper were used directly if there are no specifically noted was bought from commercial sources and the water used in this work was triple distilled (Table S1, ESI $\dagger$ ). All the cells were cultured in carbon dioxide incubator at $37^{\circ} \mathrm{C}$ containing $5 \% \mathrm{CO}_{2}$.

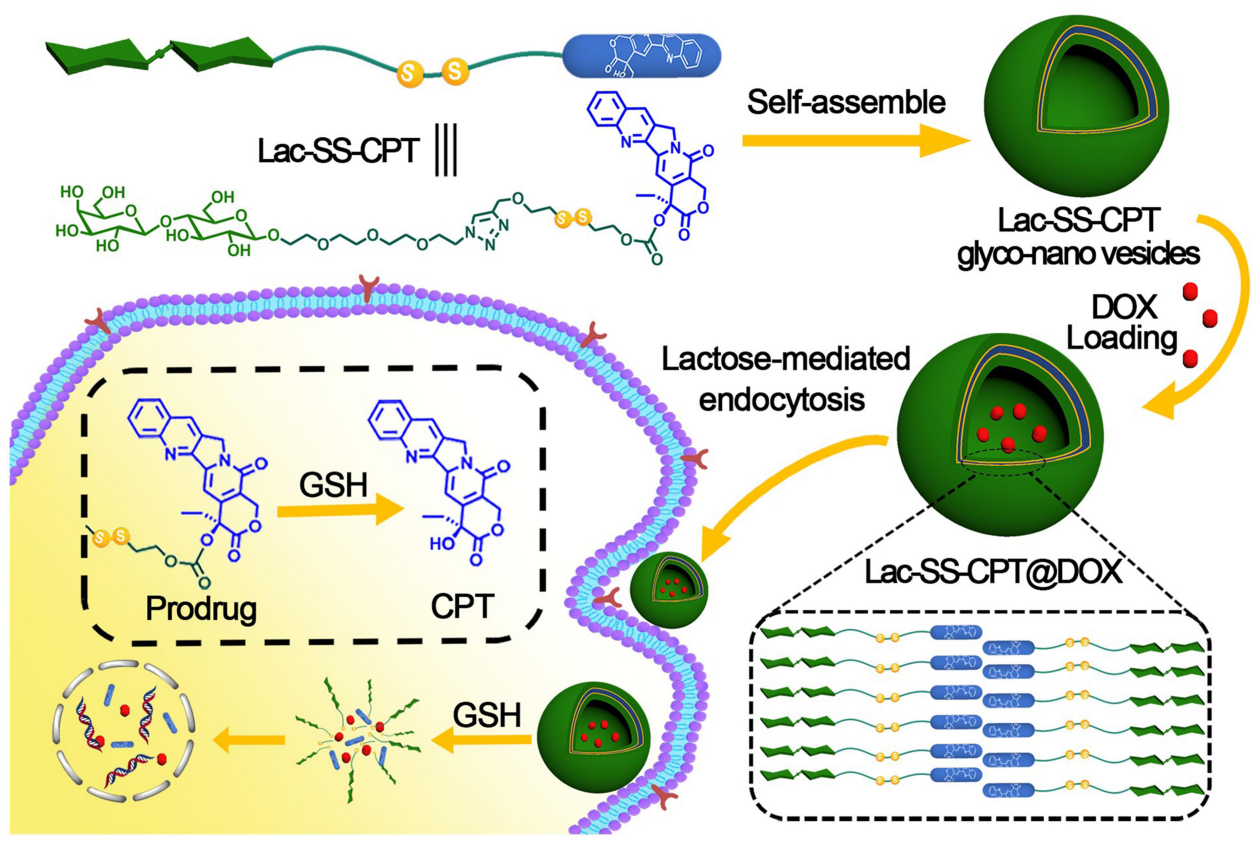

Scheme I Schematic illustration of the structure of amphiphilic prodrug Lac-SS-CPT, formation of Lac-SS-CPT glyco-nano vesicles, drug delivery, and release for cancer therapy. 


\section{Methods}

Prepare and Characterize the Lac-SS-CPT Glyco-Nano Vesicles

Lac-SS-CPT $(1.1 \mathrm{mg})$ was dissolved in $1 \mathrm{~mL}$ distilled water and ultrasonic for 30 minutes to obtain Lac-SSCPT glyco-nano vesicles $(1 \mathrm{mM})$. The same as the LacSS-CPT, Lac-SS-CPT@DOX glyco-nano vesicles (Lac-SS -CPT@DOX) were obtained by adding 1 mL 1 mM DOX aqueous solution to Lac-SS-CPT and ultrasonic for 30 minutes. Then Lac-SS-CPT@DOX was rested overnight and dialysis in the dialysis bag of molecular weight cutoff $8,000-14,000$ in water. Next, the dialysate was replaced every 2 hours until the dialysate contained no DOX. LacSS-CPT@DOX was observed and characterized by the Tyndall effect, SEM, TEM, DLS, and CAC.

\section{Release Efficiency of CPT and DOX from Lac-SS-CPT @DOX}

The release efficiency of CPT and DOX from Lac-SS-CPT @DOX was researched in $10 \mathrm{mM} \mathrm{GSH}$ solutions due to the existence of a disulfide bond. ${ }^{46,47}$ Firstly, $1 \mathrm{~mL}$ of LacSS-CPT@DOX glyco-nano vesicles was added into a dialysis bag and then dialysis was performed in $30 \mathrm{~mL}$ GSH solution of $10 \mathrm{mM}$. Secondly, $100 \mu \mathrm{L}$ of dialysate was taken out and the OD value was measured with enzyme-labeled instrument within a specified time. Lastly, the amount of drug at different times to get the release rate was calculated.

\section{Cell Culture and Cell Viability}

In this work, HepG2 (human liver cancer cells) and HL7702 (human normal liver cells) were selected to evaluate the efficacy of anticancer and reduction of side effects. HepG2 cells, HepG2-ADR cells (DOX-resistance HepG2 cells) and HL7702 cells were obtained from KeyGEN BioTECH Co. (Nanjing, China) and 1640 medium was used to culture them. Fetal Bovine Serum and Penicillin-Streptomycin were found in all the culture medium at concentrations of $10 \%$ and $1 \%$, respectively.

\section{Results and Discussion}

To obtain amphiphilic prodrug Lac-SS-CPT, hydrophilic lactose derivative 4 and hydrophobic camptothecin derivative 6 were first synthesized. Then 8 was synthesized by clicking reaction which was catalyzed by copper between 4 and 6 . The synthesis and characterization details can be seen from the ESI $\dagger$ (Supplementary Scheme S1-S3, Figure S1-S12).
Aqueous solution of amphiphilic prodrug Lac-SS-CPT was sonicated for 30 minutes to obtain the supramolecular Lac-SS-CPT glyco-nano vesicles. SEM (Figure 1A) showed a spherical nanostructure was formed. It could be observed by TEM (Figure 1B) that nanoparticles have hollow shells and it can be used to load drugs. Then, the diameter and zeta potential were analysis by DLS and the glyco-nano vesicles with $150 \mathrm{~nm}$ (Figure 1C) and -19 $\mathrm{V}$ could be found. The polydispersity of Lac-SS-CPT glyco-nano vesicles was measured to be 0.309 by DLS. Last, the CAC of the glyco-nano vesicles was measured to be $0.9 \mathrm{mM}$ with the water surface tension method (Supplementary Figure S13, ESI $\dagger$ ).

By adding GSH to the vesicular solution to investigate the GSH-responsive property of the Lac-SS-CPT glyconano vesicles. An obvious Tyndall effect (Supplementary 3-2 and Figure S14, ESI $\dagger$ ) could be observed in Lac-SSCPT aqueous solution $(100 \mathrm{mM})$ which indicated that abundant nanoparticles exist while the Tyndall effect of Lac-SSCPT glyco-nano vesicles disappeared after adding $10 \mathrm{mM}$ GSH which indicated that nanoparticles were burst. SEM showed nothing after adding GSH to the vesicular solution which was consistent with the disappearance of Tyndall effect (Figure 1D). These results all proved that the LacSS-CPT glyco-nano vesicles can burst and release the drug in GSH aqueous solution efficiently.

Then, DOX was selected to be loaded into Lac-SS-CPT glyco-nano vesicles to achieve the goal of combination treatment with CPT to improve the anticancer efficiency. After loaded into Lac-SS-CPT glyco-nano vesicles, SEM, DLS, and other measurement methods were used to observe the morphology, size, DOX loading efficiency, and release rate of Lac-SS-CPT@DOX. The surface of Lac-SS-CPT @DOX was found to be quite rough by SEM (Supplementary Figure S15) and the average diameter of Lac-SS-CPT@DOX was around 270 nm detected by DLS (Supplementary Figure S16). Last, the loading content of CPT was found to be $31 \mathrm{wt} \%$, and the encapsulation efficiency of the DOX was $78 \%$, which was because of the stable chemical structure of prodrug making it hard to destroy.

As a good drug-loaded nano vesicle, it is necessary to release active drugs in cancer cells. Therefore, it is extremely important to study the release efficiency of drug-loaded nanovesicles. As a GSH responsive group, disulfide bonds which would break in GSH solution were used in this work to make glyco-nano vesicles rupture and release drug molecules with anticancer activity. The GSH response principle of 
A
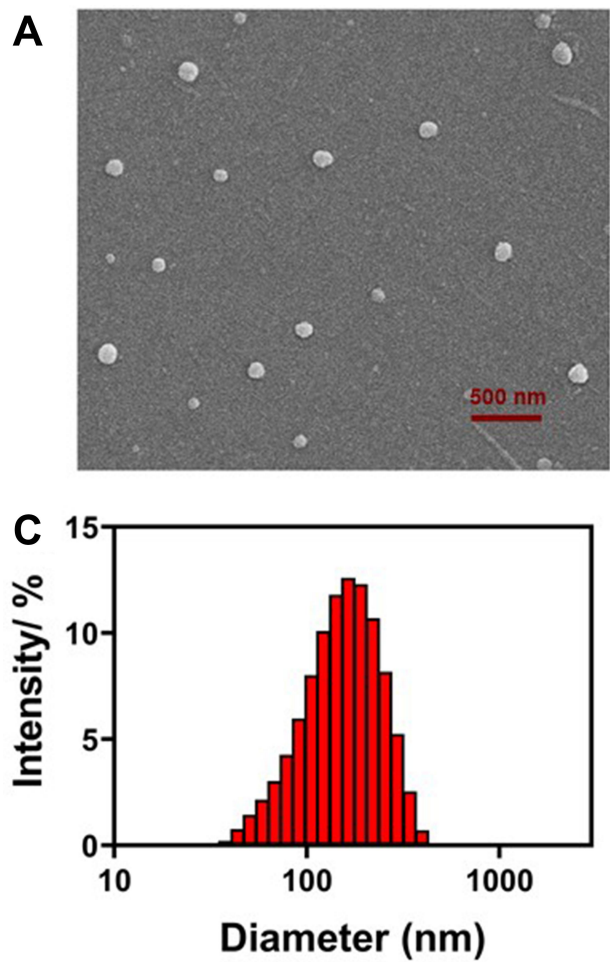

B

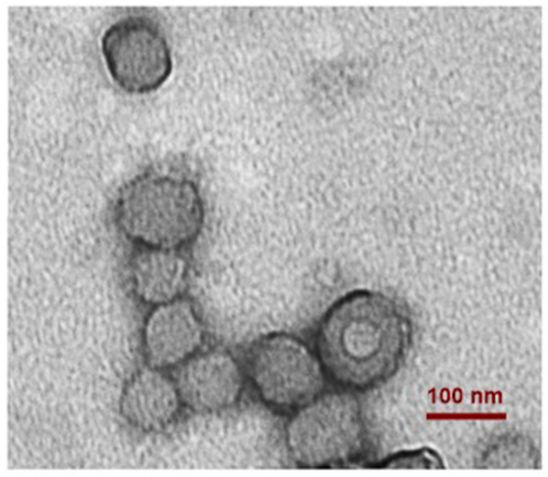

D

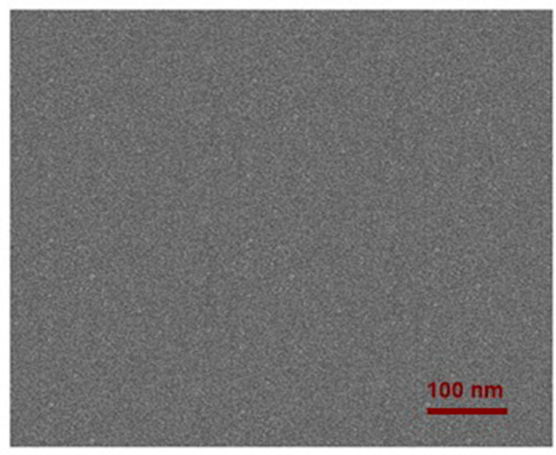

Figure I (A) SEM, (B) TEM, and (C) DLS data of Lac-SS-CPT glyco-nano vesicles based on self-assembly of amphiphilic prodrug Lac-SS-CPT; (D) SEM image of Lac-SS-CPT glyco-nano vesicles in GSH aqueous solution.

disulfide bonds is shown in Scheme 1. Firstly, in order to imitate the microenvironment of cancer cells (GSH concentration was 1-11 mM), $10 \mathrm{mM}$ GSH solution was configured. Then, the CPT and DOX release behavior from Lac-SS-CPT @DOX were investigated in $10 \mathrm{mM} \mathrm{GSH}$ and $23 \%$ and $76 \%$ of CPT were released under water and GSH aqueous solution, while $21 \%$ and $86 \%$ of DOX were released under water and GSH aqueous solution, which can be seen in Figure 2A.

Next, it is extremely important to study the targeting effect of the glycol-nano prodrug systems. HepG2 cells and HL7702 cells were chosen as target cancer cells and normal cells to study the targeting effect of different cells, respectively. Lac-SS-CPT and Lac-SS-CPT@DOX were used to culture two different cells for 4 hours with or without being preincubated with lactobionic acid (LBA). The targeting effect was analyzed by flow cytometry, where strong fluorescence intensity means more cellular uptake. Seen in Supplementary 4-1, Supplementary Figure S17 and Figure 2B, when HepG2 cells were incubated with Lac-SSCPT and Lac-SS-CPT@DOX, the fluorescence intensity (red curve) was stronger than preincubated with lactose (blue curve), which was a result from the glyco-nano vesicles

\section{A}

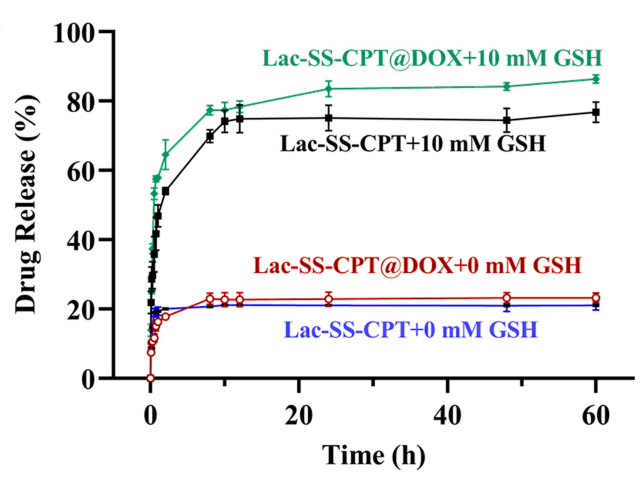

B

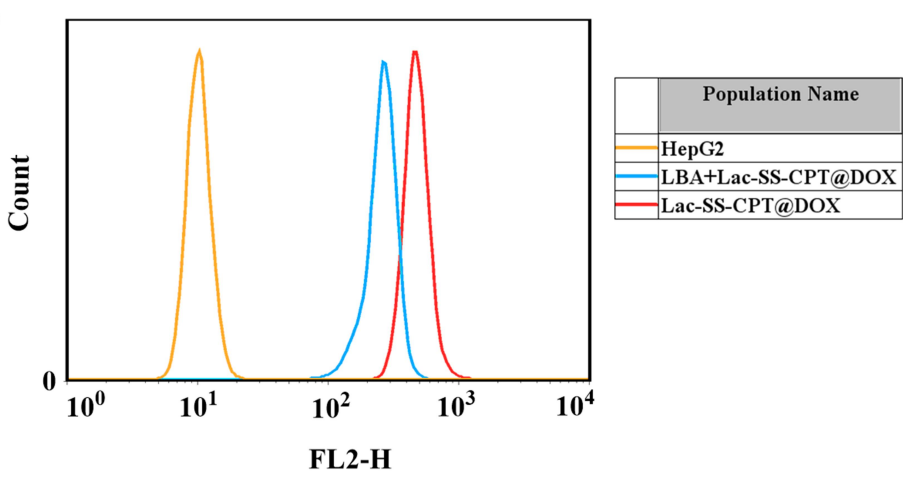

Figure 2 (A) Camptothecin and Doxorubicin release from Lac-SS-CPT and Lac-SS-CPT@DOX with 10 mM GSH concentrations at a specific time; (B). Flow cytometry analyzes the targeting effect of Lac-SS-CPT@DOX of HepG2 cells. 
targeting cancer cells and a higher uptake of glyco-nano vesicles. To further study the endocytosis of Lac-SS-CPT (a)DOX, CLSM was selected to observe the fluorescence of CPT and DOX. When HepG2 cells were cultured for 4 hours with Lac-SS-CPT@DOX, blue fluorescence could be seen with CLSM under $365 \mathrm{~nm}$ and red fluorescence could be observed under 488 nm, indicating that Lac-SS-CPT@DOX entered into the HepG2 cells (Supplementary 4-2 and Figure 3). Thus, the above results demonstrate that Lac-SSCPT and Lac-SS-CPT@DOX have good HepG2 cells targeting ability and can be applied into nanodrug delivery systems effectively.

Last but not least, the anticancer efficiency of nano vesicles is extremely important, which is directly related to the application of nano vesicles in nano-drug delivery systems. Methyl thiazole tetrazolium (MTT) assay which can effectively detect cell survival, growth, and proliferation was used to evaluate the anticancer efficiency of the Lac-SS-CPT glyco-nano prodrug system (Supplementary 4-3). Seen in Figure 4A, the cell viability of Lac-SS-CPT to HL7702 cells was higher compared with free CPT, which indicated that Lac-SS-CPT can reduce side-effects (Supplementary Figure S18a). However, the cell viability of liver cancer cells (HepG2 cells, Figure 4B and Supplementary Figure S18b) was lower when incubated with Lac-SS-CPT than incubated with free CPT. And when preincubated with lactobionic acid and then incubated with Lac-SS-CPT, HepG2 cells viability was significantly higher than Lac-SSCPT and free CPT, which indicated that the Lac-SS-CPT can target HepG2 cells and increase the toxicity.

MTT assay was used to evaluate the anticancer efficiency and combination therapy of Lac-SS-CPT@DOX, where free DOX, CPT+DOX, and Lac-SS-CPT@DOX were used to incubate HepG2 cells and HL7702 cells for 24, 48, and 72 hours. Seen in Figure 4C, compared with free DOX and CPT +DOX, the viabilities HL7702 cells with Lac-SS-CPT@DOX were higher when cultured under the same conditions. However, the viabilities were visibly lower than free DOX and CPT+DOX, where HepG2 cells were incubated with LacSS-CPT@DOX for 24, 48, and 72 hours (Figure 4D).

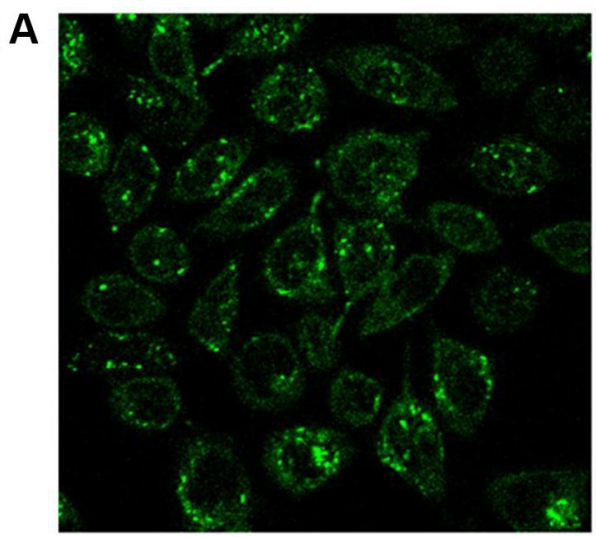

B

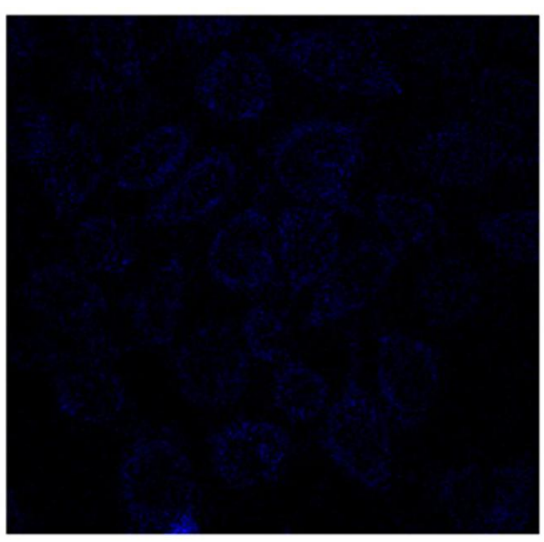

D

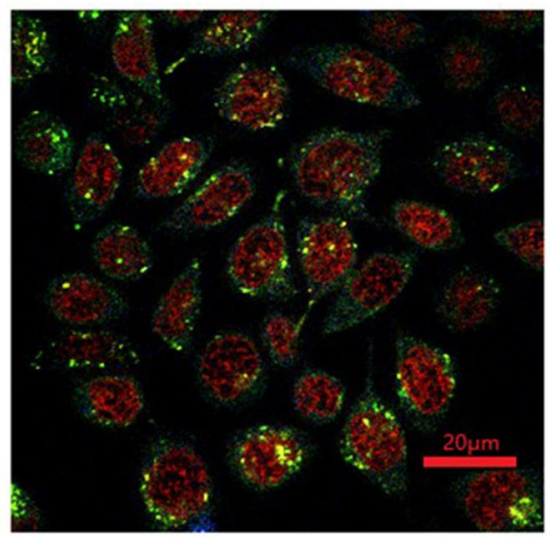

Figure 3 CLSM of HepG2 cells after incubated with Lac-SS-CPT@DOX for 4 hours, (A) Lyso Tracker Green; (B) CPT; (C) DOX; (D) merged (Mean of CPT was 33.08 and DOX was 39.74). 

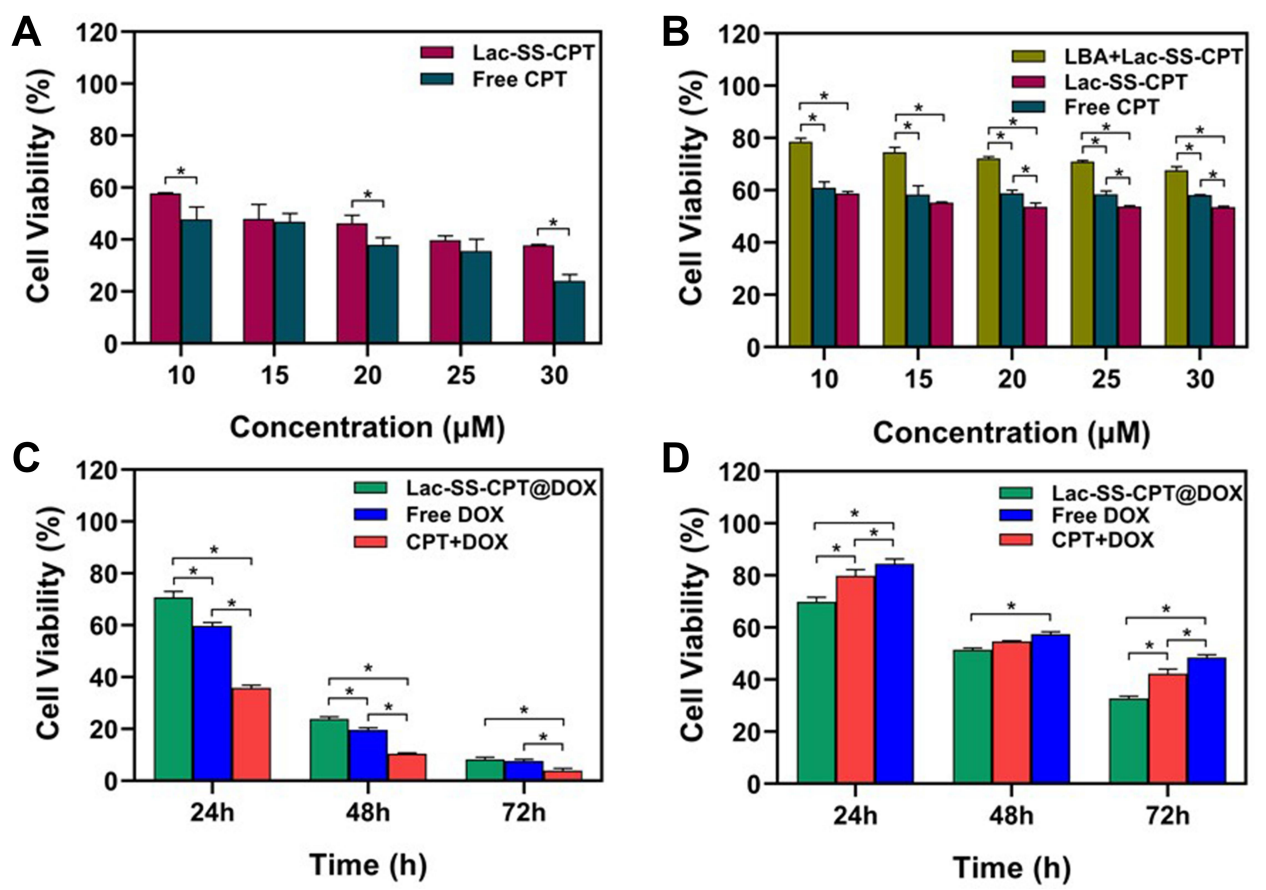

Figure 4 Cell viability of (A) HL7702 cells and (B) HepG2 cells after cultured with Lac-SS-CPT for 48 hours; Cell viability of (C) HL7702 cells (D) HepG2 cells after incubated with I $\mu$ M Lac-SS-CPT@DOX for 24,48 , and 72 hours. Statistically significant differences were observed $(* P<0.01)$.

Next, HepG2-ADR cells were selected to study the combined anticancer efficiency of CPT and DOX, where DOX, CPT+DOX, and Lac-SS-CPT@DOX were used to incubate HepG2-ADR cells for 24, 48, and 72 hours. As show in Figure 5, the viabilities of HepG2-ADR cells incubated with Lac-SS-CPT@DOX was much lower compared with free DOX and CPT+DOX, which indicated Lac-SSCPT@DOX could enhance anticancer efficiency and combine effects to cancer cells. All of the results above indicate

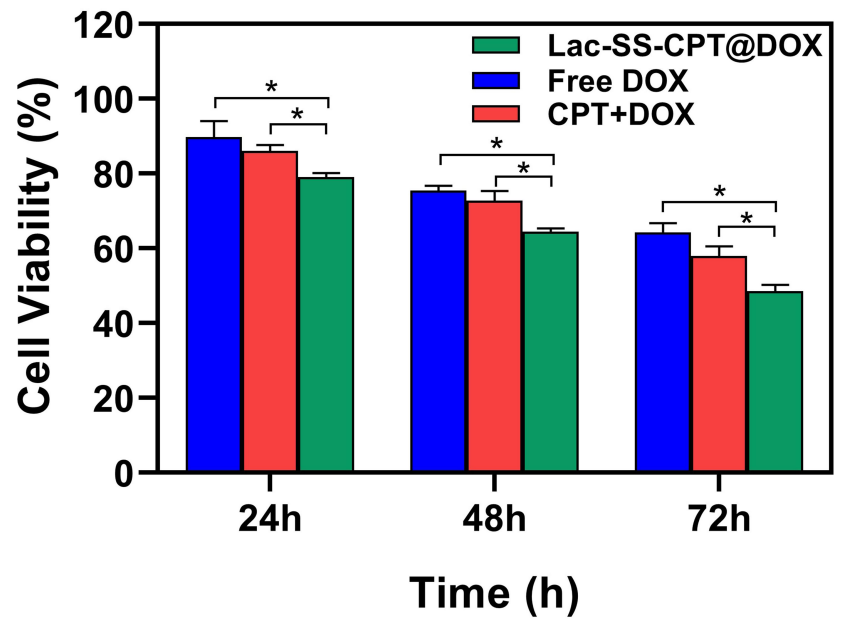

Figure 5 Comparison of the viabilities of HepG2-ADR incubated with DOX, CPT +DOX and Lac-SS-CPT@DOX at different time periods; the concentration of CPT and DOX was I $\mu$ M. Statistically significant differences were observed $(* P<0.01)$. that Lac-SS-CPT@DOX possesses many advantages, such as enhanced anticancer efficiency and combined effects to cancer cells, while reducing the side-effects to normal cells.

\section{Conclusion}

In summary, a multifunctional glyco-nano prodrug system was been successfully constructed based on the selfassembly of amphiphilic lactose modified camptothecin prodrug molecules which exhibited GSH responsiveness and cancer cells targetability. Lac-SS-CPT@DOX have high CPT loading content and its hollow can be used for antitumor drug such as DOX loading. It was verified that DOX was successfully encapsulated into the system by drug loading experiments and DOX was quickly released in $10 \mathrm{mM} \mathrm{GSH}$ aqueous solution by DOX release experiments. As expected, Lac-SS-CPT@DOX, which can target to HepG2 cells and burst under tumor microenvironment, improved anticancer efficiency to cancer cells while reducing side-effects to normal cells. Most notably, the Lac-SS -CPT@DOX which was used to incubate HepG2-ADR cells could enhance the anticancer efficiency and reduce the drug resistance of cancer cells by the combined effects. This work provides insights for rational design in the construction of a stimuli-responsive nano prodrug system for targeting drug delivery, which have great applications for combination therapy. 


\section{Acknowledgments}

This research work was supported by the National Natural Science Foundation of China (No. 21877088, 21772157, and 21572181). Specialized Research Fund for the Doctoral Program of Hebei Agricultural University (ZD201712), and Natural Science Foundation of Hebei Province (B2019204243).

\section{Disclosure}

The authors report no conflicts of interest for this work.

\section{References}

1. He WX, Hu X, Jiang W, et al. Rational Design of a New Self-Codelivery System from Redox-Sensitive Camptothecin-Cytarabine Conjugate Assembly for Effectively Synergistic Anticancer Therapy. $A d v$ Healthcare Mater. 2017;6:1700829.

2. Torre LA, Bray F, Siegel RL, et al. Global Cancer Statistics, 2012. CA Cancer J Clin. 2015;65(2):87-108. doi:10.3322/caac.21262

3. Siegel R, DeSantis C, Jemal AJ. Colorectal Cancer Statistics, 2014. CA Cancer J Clin. 2014;64(2):104-117. doi:10.3322/caac.21220

4. Cheng ZL, Zaki AA, Hui JZ, Muzykantov VR, Tsourkas A. Multifunctional Nanoparticles: cost Versus Benefit of Adding Targeting and Imaging Capabilities. Science. 2012;338:903-910.

5. Ahles TA, Root JC. Cognitive Effects of Cancer and Cancer Treatments. Annu Rev Clin Psychol. 2018;14:425-451.

6. Quinn DI, Sandler HM, Horvath LG, Goldkorn A, Eastham JA. The evolution of chemotherapy for the treatment of prostate cancer. Ann Oncol. 2017;28:2658-2669.

7. Chang YC, Lv YH, Wei P, et al. Multifunctional Glyco-Nanofibers: siRNA Induced Supermolecular Assembly for Codelivery In Vivo. Adv Funct Mater. 2017;27:1703083.

8. Vincent JV, Eric ES. Cancer Therapies Utilizing the Camptothecins: A Review of the in Vivo Literature. Mol Pharmaceutics. 2010;7:307-349.

9. Gianfranco P, Fabiana C, Lisa DV, et al. Antitumoral activity of PEG-gemcitabine prodrugs targeted by folic acid. $J$ Controlled Release. 2008;127:239-248.

10. Hu XL, Liu GH, Li Y, Wang XR, Liu SY. Cell-Penetrating Hyperbranched Polyprodrug Amphiphiles for Synergistic Reductive Milieu-Triggered Drug Release and Enhanced Magnetic Resonance Signals. J Am Chem Soc. 2015;137(1):362-368. doi:10.1021/ja5105848

11. Huang P, Wang D, Su Y, et al. Combination of Small Molecule Prodrug and Nanodrug Delivery: amphiphilic Drug-Drug Conjugate for Cancer Therapy. J Am Chem Soc. 2014;136(33):11748-11756. doi:10.1021/ja505212y

12. Chang YC, Yang K, Wei P, et al. Cationic Vesicles Based on Amphiphilic Pillar[5]arene Capped with Ferrocenium: A Redox-Responsive System for Drug/siRNA Co-Delivery. Angew Chem Int Ed. 2014;53(48):13126-13130. doi:10.1002/anie.201407272

13. Chen YY, Huang ZH, Zhao HY, et al. Supramolecular Chemotherapy: cooperative Enhancement of Antitumor Activity by Combining Controlled Release of Oxaliplatin and Consuming of Spermine by Cucurbit[7]uril. ACS Appl Mater Interfaces. 2017;9(10):8602-8608. doi:10.1021/acsami.7b01157

14. Cheetham AG, Chakroun RW, Ma W, Cui HG. Self-assembling prodrugs. Chem Soc Rev. 2017;46(21):6638-6663. doi:10.1039/ C7CS00521K

15. Aurizio ED, Sozio P, Cerasa LS, et al. Biodegradable Microspheres Loaded with an Anti-Parkinson Prodrug: an in Vivo Pharmacokinetic Study. Mol Pharmaceutics. 2011;8(6):2408-2415. doi:10.1021/ $\mathrm{mp} 200337 \mathrm{~h}$
16. Duan X, Bai T, Du JJ, Kong J. One-pot synthesis of glutathioneresponsive amphiphilic drug self-delivery micelles of doxorubicindisulfide-methoxy polyethylene glycol for tumor therapy. $J$ Mater Chem B. 2018;6(1):39-43. doi:10.1039/C7TB02817B

17. Cheng Q, Li SK, Ma YL, Yin H, Wang RB. pH-Responsive supramolecular DOX-dimer based on cucurbit[8]uril for selective drug release. Chinese Chem Lett. 2020;31(5):1235-1238. doi:10.1016/j. cclet.2019.10.020

18. Cai H, Dai XH, Wang XM, et al. A Nanostrategy for Efficient Imaging-Guided Antitumor Therapy through a Stimuli-Responsive Branched Polymeric Prodrug. Adv Sci. 2020;7(6):1903243. doi:10.1002/advs.201903243

19. Chen K, Cai H, Zhang H, et al. Stimuli-responsive polymer-doxorubicin conjugate: antitumor mechanism and potential as nano-prodrug. Acta Biomater. 2019;84:339-355. doi:10.1016/j. actbio.2018.11.050

20. He SS, Jiang YY, Li JC, Pu KY. Semiconducting Polycomplex Nanoparticles for Photothermal Ferrotherapy of Cancer. Angew Chem Int Ed. 2020;59(26):10633-10638. doi:10.1002/anie.202003004

21. Jiang YY, Li JC, Zeng ZL, Xie C, Lyu Y, Pu KY. Organic Photodynamic Nanoinhibitor for Synergistic Cancer Therapy. Angew Chem Int Ed. 2019;58(24):8161-8165. doi:10.1002/ anie. 201903968

22. Zhang HY, Zhu Y, Sun CY, et al. GSH responsive nanomedicines self-assembled from small molecule prodrug alleviate the toxicity of cardiac glycosides as potent cancer drugs. Int $J$ Pharmaceut. 2020;575:118980. doi:10.1016/j.ijpharm.2019.118980

23. Chao S, Lv XK, Ma N, et al. A supramolecular nanoprodrug based on a boronate ester linked curcumin complexing with water-soluble pillar[5]arene for synergistic chemotherapies ${ }^{\dagger}$. Chem Commun. 2020;56(62):8861-8864. doi:10.1039/D0CC04315J

24. Chen K, Liao SS, Guo SW, et al. Multistimuli-responsive PEGylated polymeric bioconjugate-based nano-aggregate for cancer therapy. Chem Eng J. 2020;391:123543. doi:10.1016/j.cej.2019.123543

25. Pan DY, Zheng XL, Zhang QF, et al. Dendronized-Polymer Disturbing Cells' Stress Protection by Targeting Metabolism Leads to Tumor Vulnerability. Adv Mater. 2020;32(14):1907490. doi:10.1002/adma.201907490

26. Zhang Y, Xu C, Yang XL, Pu KY. Photoactivatable Protherapeutic Nanomedicine for Cancer. Adv Mater. 2020;32(34):2002661. doi:10.1002/adma.202002661

27. Sun N, Zhao CY, Cheng R, et al. Cargo-Free Nanomedicine with $\mathrm{pH}$ Sensitivity for Codelivery of DOX Conjugated Prodrug with SN38 To Synergistically Eradicate Breast Cancer Stem Cells. Mol Pharmaceutics. 2018;15(8):3343-3355. doi:10.1021/acs. molpharmaceut.8b00367

28. Chen W-H, Lei Q, Yang C-X, et al. Bioinspired Nano-Prodrug with Enhanced Tumor Targeting and Increased Therapeutic Efficiency. Small. 2015;11(39):5230-5242. doi:10.1002/smll.201500920

29. Zhou T, Zhang W, Cheng DL, Tang X, Feng JF, Wu W. $<p>$ Preparation, Characterization, and in vivo Evaluation of NK4Conjugated Hydroxycamptothecin-Loaded Liposomes. Int J Nanomedicine. 2020;15:2277-2286. doi:10.2147/IJN.S243746

30. Yang L, Hong J, Di J, et al. 10-Hydroxycamptothecin (HCPT) nanosuspensions stabilized by mPEG1000-HCPT conjugate: high stabilizing efficiency and improved antitumor efficacy. Int J Nanomedicine. 2017;12:3681-3695. doi:10.2147/IJN.S134005

31. Lee BS, Amano T, Wang HQ, et al. Reactive Oxygen Species Responsive Nanoprodrug to Treat Intracranial Glioblastoma. ACS Nano. 2013;7:3061-3077.

32. Cheetham AG, Zhang PC, Lin YA, Lock LL, Cui HG. Supramolecular Nanostructures Formed by Anticancer Drug Assembly. J Am Chem Soc. 2013;135:2907-2910.

33. Gheybi H, Adeli M. Supramolecular anticancer drug delivery systems based on linear-dendritic copolymers. Polym Chem. 2015;6:2580-2615. 
34. Liu K, Jiang X, Hunziker P. Carbohydrate-based amphiphilic nano delivery systems for cancer therapy. Nanoscale. 2016;8:16091-16156.

35. Yu HL, He J, Lu Q, et al. Anti-fas antibody conjugated nanoparticles enhancing the antitumor effect of camptothecin by activating the Fas-FasL apoptotic pathway. ACS Appl Mater Interfaces. 2016;8:29950-29959.

36. Fang Y, Xue JX, Gao S, et al. Cleavable PEGylation: a strategy for overcoming the "PEG Dilemma" in efficient drug delivery. Drug Deliv. 2017;24:22-32.

37. Shen YQ, Jin EL, Zhang B, et al. Prodrugs Forming High Drug Loading Multifunctional Nanocapsules for Intracellular Cancer Drug Delivery. J Am Chem Soc. 2010;132:4259-4265.

38. Hou ML, Gao YE, Shi XX, et al. Methotrexate-based amphiphilic prodrug nanoaggregates for co-administration of multiple therapeutics and synergistic cancer therapy. Acta Biomater. 2018;77:228-239.

39. Lu YC, Song SY, Hou CX, et al. Facile fabrication of branched-chain carbohydrate chips for studying carbohydrate-protein interactions by QCM biosensor. Chinese Chem Lett. 2018;29:65-68.

40. D'Souza AA, Devarajan PV. Asialoglycoprotein receptor mediated hepatocyte targeting-Strategies and applications. $J$ Controlled Release. 2015;203:126-139.

41. Dai LL, Zhang QF, Li JH, et al. Dendrimerlike Mesoporous Silica Nanoparticles as $\mathrm{pH}-$ Responsive Nanocontainers for Targeted Drug Delivery and Bioimaging. ACS Appl Mater Interfaces. 2015;7:7357-7372.
42. Guo X, Wei X, Chen Z, et al. Multifunctional nanoplatforms for subcellular delivery of drugs in cancer therapy. Prog Mater Sci. 2020;107:100599.

43. Zhang YH, Zhang YM, Sheng XL, Wang J, Liu Y. Enzyme-responsive fluorescent camptothecin prodrug/polysaccharide supramolecular assembly for targeted cellular imaging and in situ controlled drug release. Chem Commun. 2020;56:1042-1045.

44. Lu YC, Hou CX, Ren JL, et al. A multifunctional supramolecular vesicle based on complex of cystamine dihydrochloride capped pillar [5]arene and galactose derivative for targeted drug delivery. Int J Nanomedicine. 2019;14:3525-3532.

45. Shen YQ, Jin R, Zhang B, et al. Prodrugs Forming High Drug Loading Multifunctional Nanocapsules for Intracellular Cancer Drug Delivery. J Am Chem Soc. 2010;132:4259-4265.

46. Hu XL, Hu JM, Tian J, et al. Polyprodrug Amphiphiles: hierarchical Assemblies for Shape-Regulated Cellular Internalization, Trafficking, and Drug Delivery. J Am Chem Soc. 2013;135:17617-17629.

47. Liu X, Shao W, Zheng YJ, et al. GSH-Responsive supramolecular nanoparticles constructed by $\beta$-D-galactose-modified pillar[5]arene and camptothecin prodrug for targeted anticancer drug delivery. Chem Commun. 2017;53:8596-8599.
International Journal of Nanomedicine

\section{Publish your work in this journal}

The International Journal of Nanomedicine is an international, peerreviewed journal focusing on the application of nanotechnology in diagnostics, therapeutics, and drug delivery systems throughout the biomedical field. This journal is indexed on PubMed Central, MedLine, CAS, SciSearch ${ }^{\circledR}$, Current Contents ${ }^{\mathbb{R}} /$ Clinical Medicine,

\section{Dovepress}

Journal Citation Reports/Science Edition, EMBase, Scopus and the Elsevier Bibliographic databases. The manuscript management system is completely online and includes a very quick and fair peer-review system, which is all easy to use. Visit http://www.dovepress.com/ testimonials.php to read real quotes from published authors. 